\title{
The Economic Impacts of Climate Change
}

\author{
Himatesh Cirikonda ${ }^{1}$, Vishnu Kethan Nath Gopu ${ }^{2}$ \\ ${ }^{1}$ Student at Woxsen University \\ ${ }^{2}$ Student at Woxsen University \\ ${ }^{1}$ himatesh.cirikonda_2022@woxsen.edu.in, \\ ${ }^{2}$ vishnukethan.gopu_2022@woxsen.edu.in
}

\begin{abstract}
Quantifiability of the pecuniary change regarding the climatic change is foreseen yet the ignorable fact of the century. This did not lead to a catastrophic change; however, it paved the path to the change in the governance of the conditions which led to moderate methodologies. This research contrasts directly with the immediate public discussion and greenhouse gas reduction expenditures. These forecasts demonstrate that climate change initially improves economic stability. But these advantages are declining. In the later century, the effects will be more negative. The global average effects will be equivalent in poorer economies to the health loss of a few percent of income. The marginal cost of carbon dioxide pollution is estimated at over two hundred. The social costs of carbon are quite elusive. The estimated price of $\$ 50 /$ tc is somewhat lower than in the EU but far higher than the price for carbon elsewhere for a normal discount rate. Current forecasts are incomplete, with optimistic and negative prejudices on harm costs from climate change. The indirect consequences of climate change on economic growth, large-scale destruction of habitats, low chance, the effects of global change on violence and war are among the most important of the lost impacts. The effect of climate change is troublesome from the welfare point of view because it has an endogenous population, and because policy assessments can divide impatience, risk aversion, and inequality within and within nations.
\end{abstract}

Keywords: Climate change, Economy, Greenhouse gases.

\section{Introduction}

In this paper, we are going to discuss in brief and review some of the papers on the topic, the total economic impact of climate change.

The conditions specifying the impact on the cost of the individual which is exponential is based on three major factors:

The carbon cost which is incurred on the individual 
The education of the moral responsibility an individual must depend on, which in turn should help in the reduction of the above-mentioned cost.

The least neglected and the most important form of education on which the ethics of a government-run.

\footnotetext{
$\checkmark$ The sector we are measuring in

$\checkmark$ Location

$\checkmark$ The period being considered
}

\section{Literature Review}

Two composite metrics evaluate, on average, whether the environment is comprehensive or not. The impact is calculated as a deficit of welfare benefit equal and as a percentage of income. The global medium surface air temperature increases marked by climate shifts. Estimates are the safest assumptions (Best). The standard deviation (SD), the low (low), or the upper (high) confidence interval is indicated, where appropriate. The standard deviation is indicated. This table and the IPCC table are very threefold ("Arent et al. 2014"). First, d'Arge calculations are included in this table (1979). In the second place, the Mendelsohn figures are is associated with the other table figures rather than with the average population against the change in the average temperature of the city. Thirdly, in stock exchanges and not in buying power parity dollars, Maddison should be in line with the other estimates of the table forecast.

\begin{tabular}{|c|c|c|c|c|c|}
\hline \multirow[t]{2}{*}{ Study } & \multirow[t]{2}{*}{ Warming $\left({ }^{\circ} \mathrm{C}\right)$} & \multicolumn{4}{|c|}{ Impact ( $\%$ GDP) } \\
\hline & & Best & SD & Low & High \\
\hline d'Arge 1979 & -1.0 & -0.6 & & & \\
\hline Nordhaus 1982 & 2.5 & -3.0 & & -120 & 5.0 \\
\hline Nordhaus 199| & 3.0 & -1.0 & & & \\
\hline Nordhaus 1994b & 3.0 & -1.3 & & & \\
\hline \multirow[t]{2}{*}{ Nordhaus 1994a } & 3.0 & -3.6 & & -21.0 & 0.0 \\
\hline & 6.0 & -6.7 & & & \\
\hline Fankhauser 1995 & 25 & -1.4 & & & \\
\hline Berz undated & 2.5 & -1.5 & & & \\
\hline Tol 1995 & 25 & -1.9 & & & \\
\hline Nordhaus and Yang 1996 & 25 & $-1,4$ & & & \\
\hline Plambeck and Hope 1996 & 25 & -2.9 & & -13.1 & -0.5 \\
\hline \multirow[t]{2}{*}{ Mendelsohn et al. 2000} & 2.5 & 0.0 & & & \\
\hline & 2.5 & 0.1 & & & \\
\hline Nordhaus and Boyer 2000 & 25 & -1.5 & & & \\
\hline Tol 2002 & 1.0 & 2.3 & 1.0 & & \\
\hline Maddison 2003 & 2.5 & 0.0 & & & \\
\hline \multirow[t]{2}{*}{ Rehdanz and Maddison 2005} & 0.6 & -0.2 & & & \\
\hline & 1.0 & -0.3 & & & \\
\hline Hope 2006 & 2.5 & -1.0 & & -3.0 & 0.0 \\
\hline \multirow[t]{2}{*}{ Nordhaus 2006} & 3.0 & -0.9 & 0.1 & & \\
\hline & 3.0 & -1.1 & 0.1 & & \\
\hline Nordhaus 2008 & 3.0 & -2.5 & & & \\
\hline Maddison and Rehdanz 2011 & 3.2 & -5.1 & & & \\
\hline Bosello et al. 2012 & 1.9 & -0.5 & & & \\
\hline \multirow[t]{2}{*}{ Roson and van der Mensbrugghe 2012} & 29 & -2.1 & & & \\
\hline & 5.4 & -6.1 & & & \\
\hline Nordhaus 2013 & 29 & -2.0 & & & \\
\hline
\end{tabular}


Figure 1. Impact of Global Warming on GDP.

Several financial metrics are tested for weather consequences ("Deschenes and Greenstone 2007; Barreca 2012; Hsiang and Meng 2015; Hsiang et al. 2017”). In economic terms, knowing whether the impacts are random is mostly beneficial.

Therefore, it is correctly described the economic effect of temperature. While some of these articles might otherwise say, the effects of a weather shock are different from the effect of climate change. You predict the climate when it's the atmosphere. An adjustment to make sure shocks are confined to instant reactions: place an umbrella when raining and close flood doors when flowing. By comparison, climate-change adaptation applies to capital stock changes: purchase a parachute, invest in a flood gate. In other terms, shortterm plasticity is estimated by weather studies. To put forth, weather experiments assess the short-term elasticity, but the long-term elasticity is what we are targeted at. Consequently, credible evidence for extrapolating impacts from climate change is unlikely.

To put the estimates into context, the indication that the increase of 2.5 degrees Celsius would incur the loss of income of $1.3 \%$ off the income of an average individual in any respective economy. Although the economic risk assessments of climate change do not have a basis on economic theories, current estimates use well-conceived and wellaccepted approaches. The calculations in the table are based on a multitude of processes, which are all consistent. But there are only 27 forecasts on which to make qualitative conclusions on the overall effects of climate change. It is also possible to assume that it is not clear whether climate change will lead to a net profit or social loss.

\section{Impact of Changeableness}

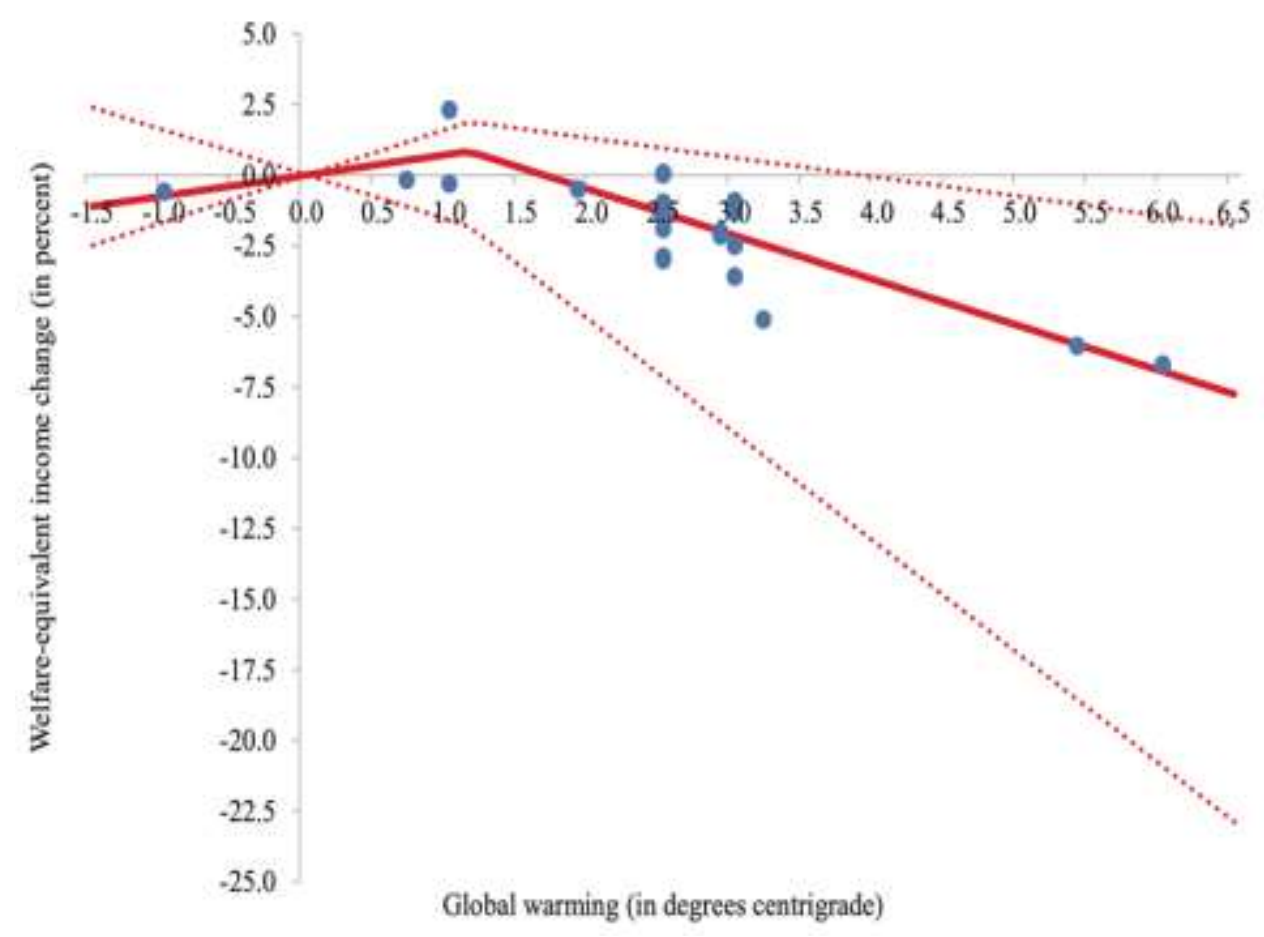




\section{Figure 1. Income Changeability due to global warming}

The unpredictability of the climate should also be considered as the estimates can always go wrong with a subtle change in any factor that governs Climate change. The dotted lines in the above figure show the normal margin of error in the table showing the trust rate of 95 percent. The real changeability may be underestimated. The initial depiction in the figure, from 0 to 3.5 does not change significantly, which is consistent with the confidence interval at face value.

From the above figures, we can derive a conclusion that negative unpredictable changes are more likely to happen than positive unpredictable changes. This applies to GHG emissions as well as to the atmosphere itself. For example, a planet that consumes a great deal of coal is simpler to picture than a world that turns quickly to wind and solar energy. Feedback to speed up climate change is more likely than feedback that dampens warming. Besides, it is typically observed that the effect of climate change is more than linear. In other words, the effects of climate change have more than doubled. However, nobody believes we can all be content with climate change. A hundred years of global warming must be reframed as well as a year of economic development, with such threats and asymmetries: It would be no less than a decade of global development if climate change were a century away.

\section{Development and Climate change Policy}

Two options will mitigate the excessive impact of climate change on the vulnerable: reducing climate change and/or reducing poverty. The connection between poverty and climate change is unclear, as we have discussed in many examples below.

\subsection{Coastal Protection}

The heavily inhabited low-lying countries of Bangladesh and the Netherlands are both at risk for water and fluvial floods. Yet Bangladesh's vulnerability to climate change is even higher. In 1850 the Netherlands began a new, broad-based deck construction program. Before that, a deck was local and rudimentary, with flooding plaguing the country daily. In 1850, the Netherlands had a little wealthier than now Bangladesh (compared to $\$ 1400$ per human a year, purchasing power parity) but the technology for constructing a dike has been improving since then. The Netherlands and Bangladesh were mostly different from one another in 1850 and 2014. Bangladesh, by comparison, is one of the world's most oppressive and badly run nations. Floods mostly impact the poor who work on the river and on the coast where land is inexpensive.

\subsection{Agriculture}

In developing countries, climate change can cut crop yields (farm-based) by up to $50 \%$. Subsistence farms yield sometimes just one-tenth of what farm versions of the same soil and environment do. The so-called yield gap is due to the lack of access to irrigated manure, high seeding, and pesticides, but due to poor roads and unhealthy land ownership, the causes for this mismatch include the lack of access to utilities and products markets. Agricultural modernization will close the gap in return and reduce the susceptibility of agriculture to climate change. 
Research from many Indian writers has shown that surface temperature patterns have risen over the last century ("Hingane et al., 1985; Srivastava et al., 1992; Rupa Kumar et al., 1994; De and Mukhopadhyay, 1998; Pante et al., 1999; Singh and Sontakke, 2002; Singh et al., 2001").

The outcomes of current GCM simulations continue to be considered unknown. The current capacity of GCM to forecast the effect on the rainfall of climate change remains unlikely. Furthermore, the forecast of severe flooding and drought is quite confusing. The predicted extent of temperature changes and precipitation in India is highly uncertain. Though climate patterns forecast a shift of -24 to 15 percent in precipitation in India before the end of the century ("Lal et al., 2001"), the regional change could differ ("Rupakumar et al., 2003"). Interannual and long-term mountain range variability and annual rainfall studies have shown that rainfall change has a statistical limit in the subcontinent ("Thapliyal and Kulshrestha, 1991; Srivastava et al., 1992"). However, analysis of past evidence shows that many places in India are seeing a warming pattern and shifts in rainfall in different areas of the country. Therefore, at this stage, it is very difficult to persuade planners and planning agencies to integrate into their schemes and farm systems the effects of climate change.

In terms of the day and night temperatures in India "Rupa Kumar et al. (1994)" demonstrated that asymmetry exists; the observed warming was largely attributed to a rise in maximum temperatures, whereas the minimum temperature in the last century remained virtually constant. "Rupa Kumar et al. (2003)" also estimate that extreme maximum and minimum temperatures around the country are likely to rise substantially because of increasing concentrations of greenhouse gases. For agriculture, this is a very significant result since the high temperature of the medium day raises the plant saturation deficit. It speeds up photosynthesis and fruit maturation (Papadakis, 1970). Maximum dry matter is produced when the temperature ranges from 20 to 30 Celsius, given that the moisture factor is not limited. Alongside high humidity, high temperatures encourage the growth of many diseases of plants. High temperatures influence the metabolism of plants as well. High temperatures at night, however, increase breathing. It promotes the growth of the stems, stolon's, shift, and berries at the cost of shooting them. It controls photosynthesis distribution between different plant organs and favors those which normally do not benefit. As a result, temperature asymmetry in day and night temperature trends can be incorporated into future research for a clearer estimate of crop production due to global warming. Extreme climate conditions, temperature abnormalities in a certain stage of development alert us to the possibility of identifying the proper management solutions without regrets. It is relatively poor and must strengthen the connection between crop pesticides and socio-economic components.

In this paper, the studies examined generally used model dynamic crop simulation to simulate production impacts. Science and technology development have built and validated dynamic cropping simulation models and been the primary instrument for the assessment of the potential impacts on climate change agriculture. To assess the risk and adaptation of agriculture to climate change, this approach is more effective. Climate changes scenarios for selected Indian subcontinent regions (turn temperature from -1\% to $+5 \%$ and plant precipitation from $-20 \%$ to +30 million) were commonly developed with GCM outcomes such as GFDL, UKMO, and GISS with climate variability.

The growing production of food grains over the last 3 decades has made India adequate and made an enormous contribution to its food security. However, the latter is now at risk, 
as demand for an ever-growing population increases. There is also a terrible situation with declining soil fertility, decreased groundwater levels, increasing salt levels, resistance to many pesticides, irrigation water quality degradation, and a rapid decline in genetic diversity of the common farming varieties. However, it is very important to preserve the natural capital. Improving the quality of organic land will increase the fertility of the soil, ensuring irrigation rates in west Indo-Gangetic Plains are used to maximize water production and other associated environmental impacts However, since it has adverse effects on rice-wheat income, there is considerable sociopolitical resistance to the implementation of this regime. In recent years, climate change prospects have sparked significant research interest to try to forecast how crop production will be carried out. The analysis aimed to provide an overview of the probable impact on food production in India of climate change.

Several studies projected increments or decreases in cereal crop yields, depending on temperature interactions and changes in $\mathrm{CO} 2$, production climate, season, and locale in India, including rice, wheat, maize, and sorghum, olive seed, and pulse crops (soybean, groundnut, chickpea, brassica, etc.). Climate effect studies have still not conducted several of Indian's key crops such as sugarcane, cotton, jute, sunflower, potato, and onion, for a better assessment of Indian agriculture's vulnerability because of climate change. But the direct impacts of climate change on 'Kharif crops are suggested by these reports, but in general 'Kharif agriculture is becoming vulnerable due to the increased impacts of severe weather conditions (monsoon, drought time, and frequency, floods, pests, and virulence). 'Rabi' crop production is comparatively riskier because the temperature increases and rainfall uncertainties are projected to rise. This will result in a decline in winter or "rabi" production unless significant adaptation takes place. A sustainability index which included economic (farm output, income, and risk) and environmental (water level, degradation of land, and biodiversity) indicators indicate the threat to agricultural production and require immediate consideration whereas, based on the uncertainty of plant models used in impact evaluation, climate change scenarios, research regions, technical change and agricultural management, climate change effects could be affected by climate changeset, an integrated assessment is of major importance to the development of potential sustainable crop cropping strategies for the different sectors of the Indian economy. Kumar and Parikh (2001) project that India's Net Revenue would decrease by 8.4 percent with $2{ }^{\circ} \mathrm{C}$ and a 7 percent rise in precipitation. It is also critical that the anticipated losses on Indian agriculture due to climate change are increased. The warm weather today for Indian farmers and the comparatively low levels of management as smaller and fewer landed marginalized farmers make up more than $60 \%$ of the overall farmers. Without a collaborative analysis in the biophysical, ecological, and socioeconomic agro-ecosystem domains, such an agricultural assessment and thus a solution to the impacts of climate change cannot be done. Although the globally integrated impact assessment models offer this type of framework, they are not appropriate for regional policy planning, since inherent inter-sectorial tensions do not provide the validation. Our integrated model simulation of assessments in which cropping systems are developed urgently; to assess the effect of environmental change in different regions in the world, the use of water and socioeconomic parameters need to be brought together. It can be built together with several stakeholders, including policymakers, agriculture, environment, climate scientists, economists, managers, industry, and the organization of farmers.

\subsection{Pandemics}


Pandemics can be brutal in any part of the world. Reducing habitat, interventions by viruses and medicines could turn a pandemic into a tropical disease. The root of disease will be transmitted by climate change and in hot weather, the viruses or the parasites are more vigorous, and the disease carriers survive in hotter and weather conditions. For instance, malaria has become prevalent in different parts of Europe and North America. In the first place, malaria is a drought. A world free of malaria can be guaranteed in investments in insecticide, bed nets, and vaccination development regardless of the air.

Analysis of the links between climate variability and infectious diseases in India based on current literature gives clear lessons for public health, study, and practice. The principle of health system resilience is particularly useful as a yard for ensuring that shocks are absorbed by the Indian health system, evolving trends of diseases respond to them, and adapting to climate change uncertainty. The resilience of Health Systems comprises the following: continuous data collection for monitoring and preparation, efficient budgetary allocation with public health funding safeguards, availability of resources including qualified staff, drugs, vaccinations, and supplies, and, above all, polycentric leadership and governance.

Resources are not sufficient for addressing the growing pressures on Indian public health infrastructure on climate variability. Given the increasing number and frequency of potential epidemic risk and the goal of epidemic response, it is either cost-effective or realistic that disease-specific responses be undertaken. Scarce public resources can also be targeted at institutional initiatives to enhance public health monitoring and overall response.

The risk among vulnerable communities also worsens socio-economic factors, which affect equity and their corresponding policies. For example, underpinning undernutrition and poverty was the acute encephalitis syndrome outbreak in 2019 that led to over 160 deaths in Bihar. The long-term policy must focus on sustainable improvement of sanitation, public investment in waste and waste management technologies, enhanced community planning, and improved health equity.

Poor communication between departments may lead to policies of various departments collaborating for cross-purpose purposes. In Delhi, for example, stubble burning is the product of water politics that reduces the differences between the agricultural seasoned in managing the water. In the neighboring farming countries, high air pollution is blamed. Also, the need for intersectoral cooperation across these Departments is reinforced: Environment, Health, Disaster Management, Urban Development, etc.

Climate adaptation includes multi-stakeholder alliances at every level, in addition to government policy. To increase awareness and action on climate change and its threats to human health, the private sector, philanthropists, researchers, and activists must all be involved. Outbreaks and behavioral changes that attempt to mitigate the spread of infectious diseases should be recruited to communities and civil society. Managing for example water and lifestyles can, despite adequate climate conditions, significantly reduce the risk of dengue and chikungunya by preventing man-mosquito contact.

The Lancet Health and Climate Change Commission in 2015 identified addressing climate change as the world's greatest health opportunity in the 21 st century 43 . The strengthening of the climatic health resilience is also a sub-set of wider healthcare strengthening. Regardless of whether illnesses are caused or not by climate change, continued improvement in the direction of human health must be the ultimate priority. 
The good news is that the same measures to enhance climatic resilience and preparedness for public health would help promote the economic and social goals of the nation. Investment in climate adaptation health systems can therefore go beyond the budgets of the health sector and cover all areas of public policy and debate.

\section{Conclusion and Discussion}

\subsection{Chances of research and policy}

The Three above examples illustrate the development and impact the climate change are closely intertwined. Though this has been debated extensively from the beginning, several studies neglected this interaction, supposing that it is constantly affected by climate change.

The deteriorating economic growth of developed countries would do more harm than good to combat climate change than the concentrated GHG decrease in rich countries. Every fifth of official development funding is being revised, while conventional aid has been revised as an ecosystem. The industrial revolution was fueled by cheap and plentiful resources and the developing countries' shortage of (reliable) power delays.

\subsection{Estimates of the carbon cost}

To achieve pledged reductions in emissions, politicians gradually use phase-out mandates instead of focusing on market-based tools such as carbon pricing. The number of advertised phase-outs of coal has increased, particularly in the power sector. Countries like France, Italy, the UK, and Finland have declared their respective programs in the Netherlands, and Portugal. Besides, the companies have announced a final closure for their coal plants in Sweden and Austria.

The following figure shows the coal phase-out notifications, which are clustered by the year of the final closure. The German charcoal exit dates of 2038 are based on a recommendation from the German charcoal exit committee, appointed to assess the issue by the government. 


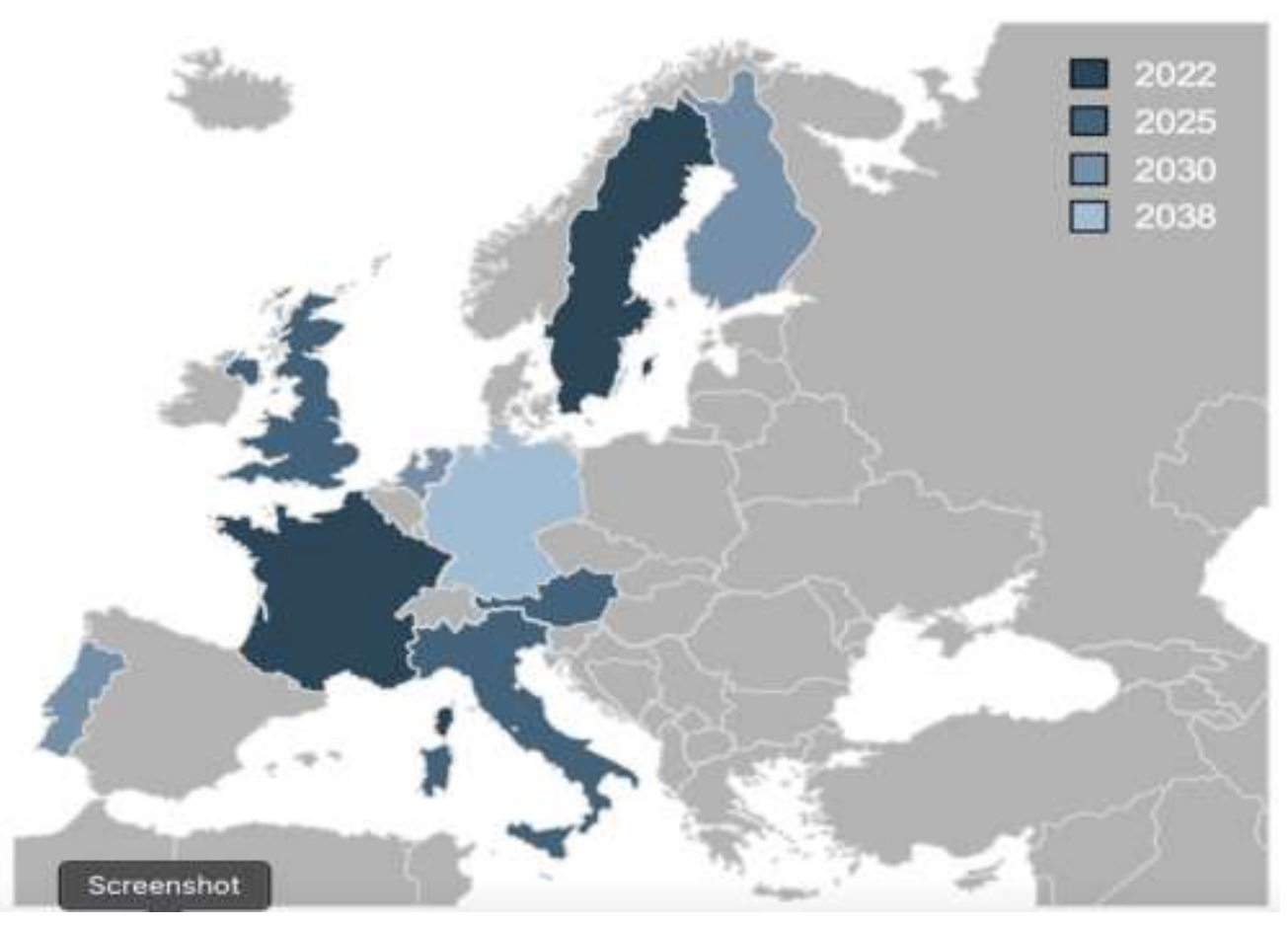

Figure 2. Coal phase-out Notifications

The difference between the theory of the efficiency of market-based instruments and the realistic tendency to announce phase-out mandates underlines the need to investigate these non-market climate policy instruments more effectively. Climate policy analysis focuses primarily on the impact and design of carbon taxes, while research into nonmarket tools like phase-out mandates has fallen well behind. There is a clear consensus on the drawbacks of coal-fired electricity generation concerning gas-fired electricity generation. The production of coal-fired electricity is usually considered to be especially dirty, and gas-fired power plant promoters emphasize that gas-fired power is less pollution intensity than charcoal-fired power generation. Therefore, the switch from fossil to gas-fueled production is supposed to help reduce carbon emissions in each unit of energy produced to decarbonize efforts.

Since the committed climate goals are a definite pollution budget, converting from coal to gas-fired electricity will stretch the time until this carbon budget has depleted without triggering warming that is incompatible with the specific warming objective. Gas-fired electricity produces less than half the volume of $\mathrm{CO} 2$ per electricity unit as opposed to coal-fired power generation, mostly because of chemicals.

Extending the period before the carbon budget has run out would provide more time for improving current or developing new zero-emission technology that can deliver energy at low cost and that is suitable for large-scale rollout. However, the overall task of decarbonizing the electricity system is ignored when solely used in charcoal and gas power production. The central issue should not be how coal-fired electricity can be reduced or how the fuel can be transferred from coal to gas. For the decarbonization route decided strategically, the main challenge is the cost-effectiveness of fuel-switching strategies. This needs not only additional production technology to be included in the response but also considering the implications of current capacity growth. On the one 
side, there is the possibility that these high carbon developments are locked up in conventional oil-fired power plants and the related generating facilities. Because of economic considerations, power plants run without premature write-downs. On the other hand, current fossil-fired power stations and the corresponding generation facility may be an integral part of the strategy for at least cost deep decarbonization.

In today's political debate and demand from climatic campaigners, auto bond pricing is a possible lever for reducing carbon emissions. The political discourse in Germany and the protest "Fridays for the future" are two recent examples. The German political discourse addresses carbon pricing as an instrument to meet the 2030 pollution reduction goal. 78 The campaign for "Fridays for the future" demands a carbon tax of $€ 160 / \mathrm{tCO} 2$ covering all GHG emissions. 79 Carbon pricing usually is carried out in practice by pollution taxes and tradable allowances. As of April 2019, 44 jurisdictions adopt one or more initiatives on carbon pricing at the federal, domestic, or sub-national level. If the 54 carbon price policies are introduced, 27 are implemented through carbon taxation and the same number of initiatives through emissions trading schemes were implemented (ETSs). The 54 carbon pricing measures, implemented in general, encompass emissions practices in the 8.0 GtCO2 range globally. This includes a fee on 2,7 GtCO2 and an ETS on 5,3 GtCO2. Overall, the covered emissions account for $14.3 \%$ of global GHG emissions. The price for both carbon-emitted tons, for the Polish carbon tax, is between $\$ 0.08 / \mathrm{tCO} 2$ and $\$ 126.78 / \mathrm{tCO} 2$ (for the Swedish carbon tax). The overall carbon price is $\$ 20.7 / \mathrm{tCO} 2$.

But there is little scientific evidence in practice that carbon pricing achieves expected pollution reductions. Sweden, for example, imposed a comparatively high carbon tax in 1991 and, although Sweden remains the world's largest carbon tax, emissions from road travel only decreased by $4.0 \%$ between 1990 and 2015 . The scant scientific evidence that carbon pricing leads to a measurable reduction of emissions leads policymakers to use alternatives that provide greater transformative capacity. For example, governments are broadly awarding incentives to accelerate the diffusion of electric cars. Subvention funding is also preferred to encourage the use in several countries of alternative sources of electricity. Conversely, the proponents of carbon pricing propose this economic instrument as the basis of climate policy. Most economists agree with the clear price of GHG emissions and recommend them.

Carbon pricing, by internalizing the externalities of these emissions of GHG in principle, lowers emissions of GHGs more cost-effectively. Carbon pricing will curb pollution from the welfare point of view at a lower expense than direct intervention, such as technological mandates or levels of efficiency. The carbon pricing literature is divided and several claims against and for carbon pricing have been made. Given the large volume of current carbon pricing literature and the latest rise of more critical literature examining the real track record of price ef-forts, an examination that recognizes all aspects of literature will bring value by finding compelling claims on each hand. Cases like those described earlier in this section of the Swedish carbon tax are contrary to the sensible agent used by the conventional economic model. Theoretical sources can nevertheless be found in behavioral economics and political science, which can clarify the observed deviation between theory and experience. Our philosophical structure is founded on the observation that the conventional economic model cannot justify why the carbon price reductions are not possible, which is why we believe that this split literature needs to synthesize. Firstly, behavioral failure is triggered by unreasonable human behavior, while an action that guides a reasonable person to the desired actions, will lead to adverse market effects. 
The behavioral economic theory gives theories about how people trigger market inefficiencies with individual desires and cognitive weaknesses to account for the Irrational actions of over seven billion homo-sapiens on the planet. Second, government failure triggered by (rational or irrational) policymakers' human actions may lead to bad policy choices and thus unwelcome market results. The conclusions from behavioral economic theory also refer here because of the human dynamics of homo politics. Political scientists also explain how bureaucratic defects limit regimes to regulate social security as maximized.

\subsection{Do we want to adjust? Temperature, work, and climate change adaptation}

This paper uses county-level payroll and day-to-day weather data to describe the effect of warm temperatures on labor and the ability of employees and companies for long-term adaptive investment. We have a considerable causal effect on the payroll during hot days, with major effects in exposed sectors like construction, transport, and production. An additional day for the US with daily peak temperatures above $95^{\circ} \mathrm{F}$ leads in exposed sectors to a decrease of -0.22 percent that year in the amount of pay per capita. Given well-known short-term wage rigidities, these effects are likely to be linked to reduced availability, commitment, and efficiency of work. These effects are not negligible, especially in exposed industries such as building and production. To illustrate that one year's average temperatures of the medium U.S. County are $+3.6 \%$ above average, 25 days above $95 \%$ are linked to an additional year for the median US county, a decline in per capita income for heavily exposed sectors of the economy by $-4.5 \%$. In highly exposed industries in the United States, we estimate about 25-40 million people. Given the number of staff exposed in many major developed economies, China and India, this figure is probably even larger worldwide. Building accounts for more than $13 \%$ of global GDP alone. This study thus demonstrates how labor productivity impacts are increased in integrated climate change assessment models, which usually expect total losses by 2100 to the order of a few percentage points of GDP. By comparing estimations of local production's short-term heat shock sensitivities amongst regions with various warm days, we interpret climate change implicitly. In one of the hottest districts of the US, for example, the effect of salary per hot day is $75 \%$ smaller than in one of the counties in the coldest quartiles, calculated by the history incidence of $96 \%$. Although several related policy unknowns have yet to be studied (e.g., potentially 23 innovation or market failures during adaptation), these estimates highlight the importance of considering potential adaptation, especially in future damage projections. At the same time, it is important to remember that adaptation can come at a significant cost that this approach does not catch. An interesting and policy-relevant field for future research is the better understanding of the scale of adaptation costs, and the nature of possible business and behavioral failures.

The paper's principal methodological message is to derive policy-related information about the possible degree of future adaptation by contrasting the local economies' shortterm temperature sensitivities, which have already adapted to different average heat exposures. In the realized performance sensitivities, cross-sectional gradients should represent Net-of-Private-Adaptation values in a different environment, a sense that comparisons are used in agriculture by Mendelsohn, etc. (1994) and others, as well as critical of causal inferences frequently associated with cross-sectional approaches. Empirically, this study forecasts local temperature sensor output and how they differ by using the cost-effectiveness revealed in local products, contrary to simulation studies that 
follow the cost and advantages of adaptation strategy by such mechanisms. This paper poses key research concerns. For example, how fair or prospective are adaptive investments made by agencies? A rational investor could choose the HVAC system for a manufacturing plant in Boston based on a weighted average of current climatic expectations. Alternatively, with its small bandwidth or lack of information, it should decide based on the intuitive meaning of the historical climate averages. Likely, decisions on worker and enterprise locations will progressively represent future climate expectations, depending on the geographical mobility of the workers and enterprises involved. Whether and how systematically education or income-based decisions differ may also be important in determining the distributive implications of climate mitigation policy and the potential for climate adaptation welfare interventions.

The welfare economies of adaptive expenditure are another set of policy-relevant matters. How much of the corresponding adaptive expenditure in private goods like house air conditioning would take on public goods at a local or international level, such as working standards, electric infrastructure (e.g., peak grid), or modern cooling technologies? An essential field of future research is to assess these and other possible market failures in light of the responses of the labor market to climate shocks. The developments that have been reported show that cost adjustment functions can be discovered using observations instead of simulations with some suppositions of the production technologies. Whilst a thorough calculation of the costs of these long-term adaptations cannot be made, similar research with rich data and/or structural estimation techniques can estimate the implicit adjustment costs that streamline observed gradients in short-run weather 24 impacts.

Finally, it is unclear if the thermal consequences and the adaptation scope are mentioned here as reflecting the expectations in other countries, mainly in developed countries. The substantial temperature-sensitivity heterogeneities in the United States, along with previous (larger) estimates of labor productivity, mortality, and agricultural production loss in developing countries, are indicative of the possibility of longer-term climate change impacts on the world's developing world more severe than previous ones. It is well known that historically air-conditioning prices have followed income growth closely and in warmer areas of the United States have a close saturation. Given a significantly lower AC penetration in many developed countries as well as in parts of Europe and East Asia, these projections indicate a significant medium- to long-term effect on labor productivity from climate change, even with rapid AC uptake.

\section{REFERENCES}

[1] "http://makeharmony1.com/vxoiw1r/4722a7-impact-of-economic-growth-onenvironment-essay". 
[2] "DEVELOPMENT AND CLIMATE CHANGE IN BANGLADESH: FOCUS ON COASTAL FLOODING AND THE SUNDARBANS by Shardul Agrawala, Tomoko Ota, Ahsan Uddin Ahmed, Joel Smith, and Maarten van Aalst “.

[3] "IMPACT OF CLIMATE CHANGE ON INDIAN AGRICULTURE: A REVIEW

R. K. MALL, RANJEET SINGH, AKHILESH GUPTA, G. SRINIVASAN and L.S. RATHORE".

[4] "Epidemics and climate change in India

Neethi V. Rao and Harini Nagendra".

[5] "Will We Adapt? Temperature, Labor and Adaptation to Climate Change.

A. Patrick Behrer, Jisung Park Harvard University". 